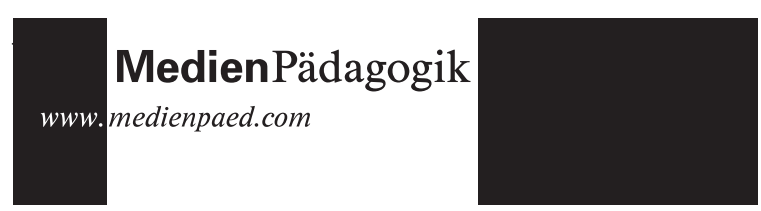

Heino Apel

17.9.2003

\section{Onlinejournal - Lernreflexionen online}

\section{Einleitung}

Lerntagebücher bzw. Lernjournale werden in der Weiterbildungspraxis eingesetzt, um Lernende darin anzuleiten, über ihren Lernprozess zu reflektieren und eine Grundlage für eine Lernberatung zu legen. Auf diese Methode wird in jüngster Zeit wieder vermehrt zurückgegriffen, weil im Kontext der Debatte zum lebenslangen Lernen die Lerner mehr Lernautonomie entfalten sollen, was eine Fähigkeit voraussetzt, Lernstrategien souverän zu handhaben und aus der Perspektive eines persönlichen Anwendungsbezugs Lerninhalte anzugehen.

Eine Internetrecherche zum Thema Lerntagebuch oder Lernjournal ergibt viele Einträge aus Schulen und Weiterbildungseinrichtungen, während eine Stichwortsuche in pädagogischen Literaturbeständen mager ausfällt. Im Angelsächsischen spricht man vom «Learning journal» und in Schweizer Links überwiegt die Bezeichnung «Lernjournal», während in Deutschland mehr von Lerntagebüchern gesprochen wird. Rambow und Nückles (2002) behaupten, dass das Konzept Lerntagebuch von Gallin \& Ruf 1990 für den Bereich des schulischen Mathematikunterrichtes entwickelt worden sei. ${ }^{1}$ Sie definieren ein Lerntagebuch als ein vom Lernenden selbst zu führendes stetiges Protokoll des eigenen Lernprozesses. Arnim Kaiser stellt ein Lerntagebuch in den Kontext metakognitiver Trainingsverfahren, wozu er auch Partnertraining, kooperatives Problemlösen und Selbstbefragungstechnik zählt. Er definiert das Lerntagebuch als ein Verfahren zur länger-

${ }^{1}$ Diese Angabe ist falsch, weil bereits 1980 Wolff-Dietrich Webler «Ein Studientagebuch als Evaluationsinstrument in der Ausbildungsforschung an Hochschulen: Konzeption u. erste Ergebnisse des Einsatzes» in Bielefeld am Interdisziplinären Zentrum für Hochschuldidaktik der Universität herausgegeben hat. fristig angelegten Analyse und Optimierung des eigenen Arbeits- und Lernverhaltens (Kaiser/Kaiser 2001).

Als didaktische Zielsetzungen nennen Rambow/Nückles

- Lernen Lernen

- Schreiben Lernen

- Motivation/Verantwortung

- Rückmeldefunktion für den Seminarleiter

Zum Lernen Lernen betonen sie wie Kaiser/Kaiser, dass es dabei eine kognitive Komponente (inhaltliche Verarbeitung des Lehrstoffes) und eine metakognitive Komponente gibt, durch die sich die Lernenden selbst beobachten und ihr eigenes Verstehen kontrollieren und über die eignen Lernstrategien reflektieren.

Motivation entsteht nicht automatisch, erst durch ein Reagieren der Seminarleitung auf eigenverantwortete Beurteilungen durch die Lernenden steigert sich das Interesse an der Mitgestaltung des Lernprozesses. Die Rückmeldung durch die Seminarleitung betrifft nicht nur eine Beratungsfunktion, sondern sie wird als wesentlich dafür erachtet, dass die Lernenden ihr Journal auch anerkannt wissen wollen, damit es nicht als Selbstbeschäftigungstherapie missverstanden wird.

Rambow/Nückles empfehlen das Journal zur Pflicht zu machen, genaue Angaben zum Umfang festzulegen und eine schriftliche Anleitung über den Sinn und die erwarteten Leistungen zu geben. Zur Anleitung empfehlen sie Leitfragen oder auch ein Raster, das von den Lernenden auszufüllen ist. Den Zeitaufwand für Lernende und Lehrende schätzen sie hoch ein und sehen hier auch eine Herausforderung sorgfältig abzuwägen, wann ein Journal Sinn macht und wann nicht.

In angelsächsischen Internetquellen zum «learning journal» findet man meist Gebrauchsanweisungen, wo dem Lerner eindeutig beschrieben wird, auf welche Fragen er wann und mit welcher Ausführlichkeit reagieren soll. Es geht in der Regel um die Metaebene des Lernens, d. h. es soll über Verständnisprobleme, Neuigkeitsaspekte, Lernfortschritte und persönliche Positionierung reflektiert werden, wobei das Lernjournal nicht mit normalen Seminaraufzeichnungen zum Lehrstoff verwechselt werden soll. Man vergleiche folgende Quellen:

〈http://www.leeds.ac.uk/sddu/assess/journal.htm〉 (Bestimmungskriterien für ein Onlinejournal) 
〈http://www.rcmp-learning.org/docs/ecdd0074.htm〉(Mechanismen eines Lernjournals im Sinne von Selbstevaluation des Lernprozesses) 〈http://www.lancs.ac.uk/staff/gyaccp/374/learning\%20journal.htm〉 (Eine detaillierte Anleitung eines Lernjournals für die Lerner eines Geografiekurses an der Lancaster University von Chris Park) 〈http://www.arrowhead.lib.mn.us/certification/journal.htm〉 (Keeping a Learning Journal als Teil eines Zertifizierungsprozesses) 〈http://www.cf.ac.uk/hisar/modules/HS5301/learnjournal.html〉 (Beschreibung eines Lernjournals und seines Stellenwertes im Assessment)

\section{Möglichkeiten von Online Lernjournalen}

In der zahlreichen Literatur zu «virtuellen» Seminaren findet man keine Hinweise zum Umgang mit netzgestützten Lerntagebüchern bzw. Journalen. Überhaupt wird insgesamt relativ viel zur tutoriellen Begleitung und zur virtuellen Moderation geschrieben, zum eigentlichen netzgestützten Lernen bzw. zu einer entsprechenden Didaktik gibt es bislang wenig Untersuchungen (vgl. z. B. Lehmann/Bloh 2002; Apel/Kraft 2003).

Wenn wir im Folgenden von netzgestütztem Lernen sprechen, dann sind damit Seminarformen gemeint, die nicht nur zur Lernverwaltung das Internet benutzen, und die auch nicht nur materialbasiert, d. h. zur Durcharbeitung von webbasierten Trainingseinheiten dienen, sondern bei denen in hohem Masse in einer netzgestützten Lernumgebung über Lerninhalte kommuniziert wird.

Folgende Befunde netzgestützten Lernens legen eine Begleitung durch Lerntagebücher nahe, die zur Lernreflexion und Metakognition anregen:

- Beim netzgestützten Lernen ist im allgemeinen die Selbststeuerungskomponente höher als beim Präsenzlernen, d. h. besonders unerfahrene Lernende brauchen mehr Anleitung (Reflexionsanstösse), mehr Lernberatung.

- Online-Lernende stehen nicht in leiblichem Kontakt mit den Lehrenden und Mitlernenden, so dass die Chancen, im Lernprozess orientierende, über das rein Fachliche hinausgehende Hinweise einzuholen gegenüber «face-to-face» Lernenden geringer sind. (Z. B. Pausengespräche oder auch nonverbale Kontakte in Präsenzseminaren über die Wertigkeit des Stoffangebotes, über den Sinn der Beispiele, über die Verständlichkeit des Angebotes sind wichtige persönliche Ergänzungen für den Verständnis- und Orientierungsprozess der Lernenden.)

- Eine weitere Schwierigkeit des Online-Lernens besteht in der Heraus- forderung, z. B. bei Forendebatten Kohärenz zu stiften. D. h. die Lernenden müssen aus der Beitragsfülle das für sie und ihren Lernfortschritt wichtige destillieren, sie müssen selbst dabei bewerten und gewichten. (Apel 2003).

Diese Defizite gegenüber dem Präsenzlernen können mit angeleiteten Reflexionsaufgaben durch ein Onlinelernjournal abgebaut werden. Wobei ein Tutor mit den Journalaufzeichnungen gezielte Lernberatung betreiben kann.

Ein weiterer Nutzungsvorteil besteht darin, dass die persönliche Beratung über das Lernjournal ein bindendes Element für die Lernenden, das ihnen eine persönliche Geborgenheit im ansonsten als relativ abstrakt und rückmeldungsarm empfundenen Lernraum gibt. Dabei sollte ein Onlinejournal so organisiert sein, dass die Einträge über eine persönliche Plattform geschehen, die jeweils nur dem einzelnen Lerner und dem Moderator zugänglich ist.

\section{Probleme}

Kommunikationsorientierte Online-Seminare, in denen von den Lernenden eine hohe Forenaktivität beim Diskussionsprozess zu den Seminarinhalten abverlangt wird, werden in der Regel von den Lernenden als zeitintensiv im Vergleich zu Präsenzseminaren empfunden. Wenn sich dazu eine zusätzliche Reflexionsaufforderung gesellt, auch wenn diese auf einer persönlichen Plattform verläuft, wird das als eine zusätzliche Arbeitsbelastung empfunden, die u. U. auch für Verwirrung sorgen kann. Im Seminar-Forum muss der Lernende bereits einen hohen Reflexionsgrad zum Lerngegenstand einbringen, und gleichzeitig soll er eine persönliche Reflexion auf «seiner» Plattform leisten. Die Seminarleitung muss deshalb Selbstreflexionsaufforderungen für das Onlinejournal sehr dosiert und nur an herausragenden inhaltlich begründbaren Stellen platzieren. Besonders in den angelsächsischen Internetquellen wird dargelegt, dass das Lernjournal Teil der Beurteilung für das Kurszertifikat ist, womit der «Mehraufwand» in die Beurteilung einfliesst.

\section{Alternativen}

Als Alternative zum persönlich orientierten Lernjournal kann man in Phasen des Online Forums Metareflexionen und persönliche Bedeutungskontexte durch entsprechende Fragen «plenar», d. h. im offenen Forum abhandeln. Auf diese Weise werden ebenfalls Lernreflexionen ausgelöst 
und Anstösse gegeben, die Lerninhalte lernbiografisch zu verorten und es kann Raum geschaffen werden, über das Lernen zu reflektieren. Die offene Behandlung von Lernreflexionsfragen hat den Vorteil, dass keine als zusätzlich empfunden Aufgaben für die Lernenden entstehen. Der Nachteil dürfte darin liegen, dass das Plenum unvermeidlich Gruppenaspekte und weniger ganz persönliche Einstellungen thematisiert. Diejenigen, die die Reflexion dringend brauchen würden, werden sehr wahrscheinlich passiv bleiben und allenfalls die Lernreflexion der anderen verfolgen.

Eine andere Möglichkeit der Integration selbstreflexiver Beiträge besteht darin, z. B. in Gruppenarbeitsphasen einem Gruppenteilnehmer den Auftrag zu geben, ein Gruppenlerntagebuch zu schreiben, das mit vorgegebenen Fragen strukturiert werden kann. Diese Form vermeidet ebenfalls den «Überlastungseffekt» und gibt zugleich die Möglichkeit eines «Spiegels» der Gruppenarbeitsweise für Teilnehmende und Moderator. Im folgenden wird ein Konzept vorgestellt, wie man ein Onlinejournal in eine Lernplattform installieren kann. Ob dieser Ansatz trägt und welchen Mehrwert er im Vergleich zur Mehrbelastung er für die Lernenden und Moderierenden bringt, muss noch erprobt werden.

\section{Konzept für ein Onlinejournal}

Das Onlinejournal wird über ein Forum organisiert, das passwortgeschützt jeweils nur einem Lernenden zugänglich ist. Diese Technik dürfte problemlos auf den gängigen Lernplattformen möglich sein. Auf diesem persönlichen Forum erhält der Lernende vorbereitete Reflexionsfragen, er trägt darin seine Reflexionen ein, und er kann in diesem Forum persönliche Fragen an den Moderator stellen. Wenn ein Eintrag eines Lerners erfolgt, erhält der Moderator eine Mail, so dass auf akute Anfragen auch akut reagiert werden kann.

Die didaktische Feinstruktur eines Onlinelernjournals hängt wie im präsenten Fall sehr von dem Lehrstoff, und hier insbesondere von der Art der installierten Lernumgebung auf der Lernplattform, von den Lernzielen, von den Vorerfahrungen der Zielgruppe, etc. ab.

Als Konzepte sind mit zunehmender Planungsstringenz denkbar:

- ein relativ vorgabefreies Begleitjournal, dass die Lernenden mit relativ wenigen Reflexionsfragen nötigt, zu bestimmten Aspekten (z. B. Kognition, Prozess, Biografisches) reflexiv Stellung zu beziehen. Dabei kann der Umfang über eine Mindestanforderung den Lernenden frei gestellt werden
- ein inhaltlich zielgerichtetes Lernjournal, bei dem die Lerner zu wesentlichen Lernzielen Stellung beziehen sollen, so dass die über das Journal initiierte Metakognition wesentlicher Teil des Vermittlungsprozesses wird. Diese Vorgehensweise ist insbesondere nutzbringend, wenn das Seminar den Anspruch des «Lernen Lernens» und der Vorbereitung auf selbstorgansiertes Lernen dienen soll. In diesem Fall sollten präzise Fragen und genaue Erwartungen über Abgabetermine und Umfang angegeben werden

- ein auf Lernbegleitung und Lernberatung zielendes Journal, das insbesondere in individualisierten Lernphasen des Onlineseminars wirksam sein muss, weil hier die ständige Präsenz eines Moderators auf dem gemeinsamen Forum fehlt, bzw. reduziert ist. In diesem Falle muss bei der Planung des Journals und seiner Interventionsschritte zur Lernberatung sorgfältig ein möglicher Lernprozess der Lernenden antizipiert werden.

Als Beispiel und Anregung für mögliche Realisierungen sind folgend Bausteine vorgestellt, die einen Einführungsblock (Erklärung, was das Journal soll), und entsprechend zu den im Seminar geplanten Sequenzen jeweils zugehörige Journalsequenzen beschreiben.

Wenn eine Lernende ihr Onlinejournal-Forum betritt, erhält sie z. B. folgende Information:

\section{Hallo, Frau Meier, willkommen im Online Lernjournal}

Dieses, nur Ihnen und dem Moderator zugängliche Forum, soll Ihnen helfen, beim online Kommunikationsprozess die Übersicht $\mathrm{zu}$ behalten, Ihre wesentlichen Erkenntnisse und Fragen zu dokumentieren, und persönliche Bezüge zum Kommunikationsgeschehen herzustellen. In erster Linie dient es Ihrer Selbstvergewisserung Ihres Lernprozesses, sie können es aber auch zur Lernberatung durch den Moderator nutzen, an den Sie in diesem Forum Fragen formulieren können, oder der zu Ihren Darstellungen gegebenenfalls Anregungen äussert.

Im linken Fenster finden Sie schon vorgefertigte (oder im Prozess erst jeweils aktuell formulierte) Fragestellungen zu den einzelnen Abschnitten des Seminars, auf die Sie jeweils am Ende des entsprechenden Seminarabschnittes unter dieser Rubrik (im rechten Fenster) antworten sollten.

Wenn Sie einen von diesen Reflexionsfragen unabhängigen 
Aspekt äussern wollen, tragen Sie den im linken Formularfeld unten ein.

Um Ihren Arbeitsaufwand in Grenzen zu halten, genügen stichwortartige, knappe Beiträge, die als persönliche Merkposten des Seminarfortganges zu betrachten sind. Sie sollten allerdings spätestens am Montag nach Abschluss eines Themenblockes Ihren Kommentar ins Forum schreiben. Wenn Sie sich am Schluss des Seminars Ihre Kommentare herauskopieren und in einer Datei ablegen, sollten Sie damit auch eine gute Erinnerungsgrundlage für das Seminar als persönliches Dokument haben.

Denken Sie daran, dass das Onlinejournal kein Ersatz für eine inhaltliche «Seminarmitschrift» sein soll, sondern wirklich eine Reflexion über die Seminarinhalte und den stattgefundenen Lernprozess ein soll.

Das hier beschriebene Forum, in dem das Onlinejournal realisiert wird, ist auf dem Monitor zweigeteilt, d. h. im linken Fenster befindet sich die «Baumstruktur» des Journals, und im rechten Fenster sieht man das jeweils geöffnete Statement (vgl. Abb. 1).

Zum Einführungsblock können z. B. folgende Reflexionsfragen formuliert werden, die eine Lernende am Ende des Blockes (im rechten Fenster des Screenshot Onlinejournal) zu beantworten hätte:

\section{Überlegungen zum Einführungsblock}

1. Was bedeutet die Lernform «Forum〉 für Sie? Skizzieren Sie stichwortartig, welche Unterschiede Sie zum Präsenzlernen empfinden.

2. Reflektieren Sie Ihre Zeitorganisation. (Häufigkeit, Dauer, Aktivitätsform [nur lesen? Notizen machen?])

3. Nachdem Sie die anderen Selbstdarstellungen gelesen haben, charakterisieren Sie Ihre eigene.

4. Weitere Bemerkungen?

[antworten Sie im unteren Feld mit Bezug auf jede Nummer (z. B. zu 1.: ....; zu 2.: .. ; etc.)]
Die Fragestellung zum ersten Themenblock könnte wie folgt lauten:

\section{Zum Themenblock 1}

1. Skizzieren Sie Inhaltslinien aus der Forumsdebatte, die Ihnen wichtig erschienen. (Inhaltsreflexion)

- war Ihnen etwas neu?

- ist etwas unklar geblieben?

- welche Vorstellungen haben Sie selber eingebracht?

- haben Sie abweichende Einschätzungen?

2. Wie erschliessen/produzieren Sie Inhalte der Forumsbeiträge?

\section{(Prozessreflexion)}

- wie finden Sie für sich den 〈roten Faden〉?

- nach welchen Kriterien werden Sie selbst aktiv?

- wie generieren Sie eigene Wissensbeiträge zum Forum?

3. Sind Sie als 〈Baumeister $>$ am Forum zufrieden mit sich?

\section{(Reflexion der Arbeit)}

- wie konnten Sie sich einbringen?

- gibt es alternative Handlungslösungen für Ihre Aktivität

- sind Sie mit der Resonanz, dem Prozessfortgang zu Ihren Beiträgen zufrieden?

4. anderes?

(Antworten Sie bitte mit Bezug auf jede Nummer (z. B.: zu 1.: ...; zu $2 .:$...; etc.)

Reflexionsaufforderung zur Gruppenarbeit:

\section{Überlegungen zur Gruppenarbeit}

1. Welche Ergebnisse (stichwortartig) erbrachte Ihre Gruppe?

(Inhaltsreflexion)

- War Ihnen und der Gruppe die Fragestellung klar?

- Welche Anregungen haben Sie von anderen TN erhalten?

- Welche Ergebnisse (Stichworte) erbrachten die anderen Gruppen?

- Was hat das Resümé im Plenum zur weiteren Inhaltsfindung beigetragen?

- Welche neuen Erkenntnisse haben Sie dabei gewonnen? 
2. Wie verlief die Arbeitsteilung und Kommunikation in der

Gruppe? (Prozessreflexion)

- Moderationsrolle? Zeitmanagement?

Selbst- und Kollegen-Disziplin?

- welche Anstösse hat die engere Gruppenkommunikation erbracht (Chat/e-Mail?)

- was lässt sich in virtueller Gruppenarbeit verbessern? (Techniken der gemeinsamen Dokumentbearbeitung?)

- waren die Anregungen (Links, Dateien, Literatur) des Seminars ausreichend? Verbesserungsvorschlag?

3. Atmosphäre der Gruppe? Eigene Positionierung?

(Arbeitsreflexion)

- Spass/Schwierigkeiten in der Gruppenarbeit?

- eigene Rolle (Moderator, Zuträger, Anreger, Trittbrett, ..)?

- falls Sie wenig aktiv waren - warum?

- falls Sie sehr aktiv waren - warum?

- geben Sie sich eine Note bei der Gruppenmitarbeit.

Reflexionsaufforderung zum Abschluss:

\section{Schlussbetrachtung}

Skizzieren Sie (in Stichworten) die inhaltlichen Highlights des

Online-Seminars. (Inhaltsreflexion)

- was ist Ihnen neu, welche Vorstellungen haben sich verfestigt?

- welche Fragen sind offen geblieben?

- ergeben sich Perspektiven für das weitere Studium, für die weitere Praxis?

- was hätten Sie sich anders gewünscht?

Ist das Onlinelernen (rückblickend) eine sinnvolle Lernform für

\section{Sie? (Prozessreflexion)}

- welche Techniken/Methoden im Seminar haben Ihnen

besonders zugesagt - warum?

- was waren eher hinderliche Faktoren für Ihr persönliches Lernen?

- beurteilen Sie Aufwands- und Ertragsverhältnis

Waren Sie mit sich im Onlinelernprozess zufrieden?

(Arbeitsreflexion)
- Glauben Sie, dass präsente Lernformen Ihnen mehr oder weniger Erkenntnisse vermittelt hätten?

- Haben Sie Ihr selbst gestecktes Ziel (ihre Erwartung zu Beginn) erreicht?

- Was würden Sie beim nächsten Onlineseminar anders machen?

- Geben Sie sich eine Note für Ihre Gesamtpräsenz im Seminar

Die Abbildung 1 zeigt einen Screenshot eines Forums zu Beginn eines Onlineseminars, bei dem der Moderator (für alle Teilnehmer) Reflexionsfragen für jeden Block vorbereitet hat. Erst wenn die Teilnehmenden im Ablauf ihre Rückmeldungen eintragen, muss die Seminarleitung entscheiden, ob sie beratend tätig wird (also Antworten zu den Rückmeldungen verfasst), oder ob sie die TN-Antworten als Ausdruck hinreichender〈Lernstabilität〉 und hinreichender reflexiver Durchdringung empfindet.

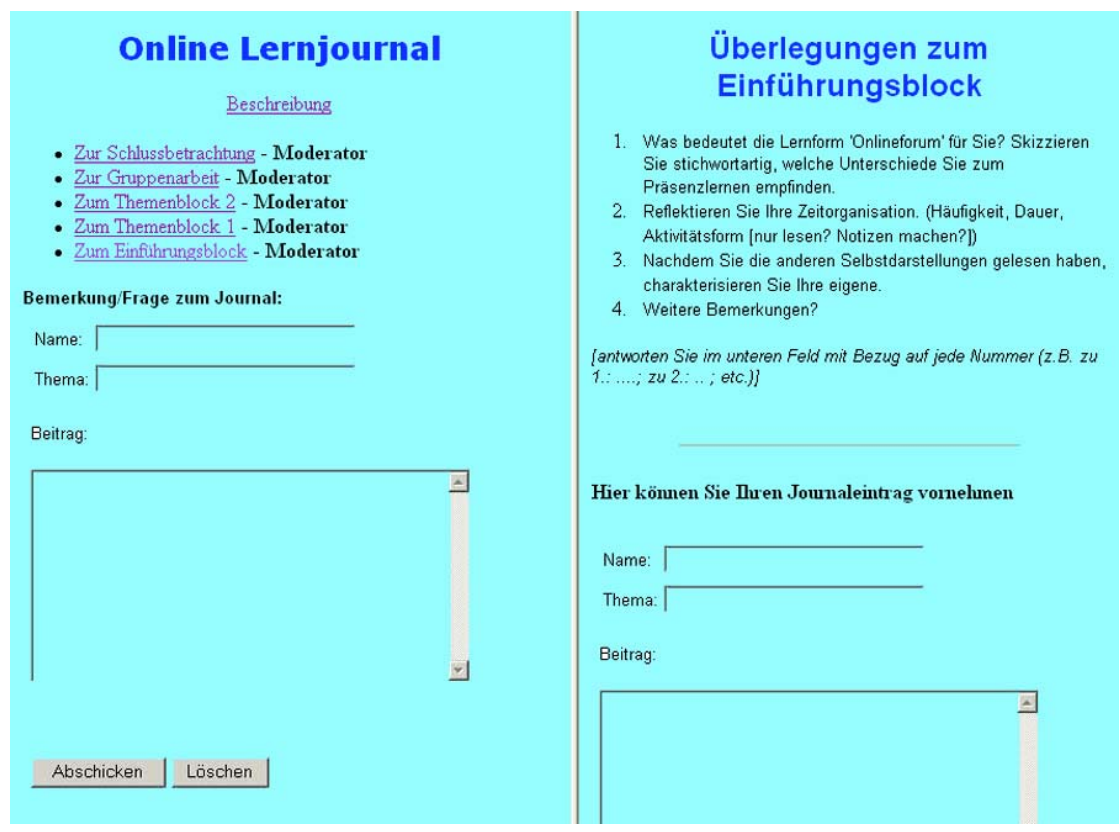

Abb.1: Screenshot eines zweigeteilten Forums (links threads, rechts Inhalte) für ein Onlinejournal 


\section{Literaturverzeichnis}

Apel, Heino. «Das Forum als zentrales Instrument asynchroner Onlineseminare». In: Online lehren. Hrsg. von Apel, Heino; Kraft, Susanne. Bielefeld 2003.

Apel, Heino; Kraft, Susanne. (Hrsg.): Online lehren. Bielefeld 2003.

Lehmann, Burkhard; Bloh, Egon. (Hrsg.): Online-Pädagogik. Hohengehren 2002.

Kaiser, A.; Kaiser, R.. Lerntagebuch und Selbstbefragung als metakognitive Studientechniken. Fernuniversität Hagen, Orientierungsstudium «Gründer werden?» 2001.

Rambow, R. Nückles, M.. «Der Einsatz des Lerntagebuchs in der Hochschullehre» In: Das Hochschulwesen, 50, 3/2002, S. 113-120.

Webler, W.-D. Ein Studientagebuch als Evaluationsinstrument in der Ausbildungsforschung an Hochschulen: Konzeption u. erste. Ergebnisse des Einsatzes, Interdisziplinäres Zentrum für Hochschuldidaktik der Universität Bielefeld 1980. 